\title{
Effect of teledentistry on the oral health status of patients undergoing fixed orthodontic treatment at the first three follow-up visits
}

\author{
Elahe Soltanmohamadi Borujeni ${ }^{1, A, F}$, Fatemeh Sarshar ${ }^{2, B, C}$, Maryam Nasiri, ${ }^{3, B}$, Samaneh Sarshar ${ }^{2, E}$, Leila Jazi ${ }^{4, B-E}$ \\ ${ }^{1}$ Department of Orthodontics, School of Dentistry, Qom University of Medical Sciences, Iran \\ 2 Orthodontist, Private Practice, Tehran, Iran \\ ${ }^{3}$ Orthodontist, Private Practice, Mashhad, Iran \\ ${ }^{4}$ Department of Orthodontics, School of Dentistry, Tehran University of Medical Sciences, Iran \\ A - research concept and design; $\mathrm{B}$ - collection and/or assembly of data; $\mathrm{C}$ - data analysis and interpretation; \\ $D$ - writing the article; $E$ - critical revision of the article; $F$ - final approval of the article
}

Address for correspondence

Leila Jazi

E-mail: leilajazi@yahoo.com

Funding sources

None declared

Conflict of interest

None declared

Received on December 11, 2020

Reviewed on February 26, 2021

Accepted on March 22, 2021

Published online on September 13, 2021

Cite as

Soltanmohamadi Borujeni E, Sarshar F, Nasiri M, Sarshar S, Jazi L. Effect of teledentistry on the oral health status of patients undergoing fixed orthodontic treatment at the first three follow-up visits. Dent Med Probl. 2021;58(3):299-304. doi:10.17219/dmp/134750

DOI

$10.17219 / \mathrm{dmp} / 134750$

Copyright

๑ 2021 by Wroclaw Medical University

This is an article distributed under the terms of the

Creative Commons Attribution 3.0 Unported License (CC BY 3.0)

(https://creativecommons.org/licenses/by/3.0/).

\begin{abstract}
Background. As the prevalence of such oral diseases as dental caries and periodontal problems increases during fixed orthodontic treatment, patient education is an essential aspect of any such treatment. Two methods have been proposed for this purpose: direct education in a clinic (the conventional method); and education using social media, such as the Telegram application (a type of teledentistry).

Objectives. The aim of this study was to evaluate the effect of teledentistry as an educational tool on the oral health status of patients undergoing fixed orthodontic treatment at the first 3 follow-up visits.
\end{abstract}

Material and methods. Sixty participants were enrolled from patients whose fixed orthodontic treatment was to begin at a dental clinic in Tehran, Iran. They were randomly assigned to one of 2 trial arms. At the end of the $1^{\text {st }}$ appointment, one of the groups was educated in person about maintaining oral hygiene during treatment, and an educational clip was sent to the members of the other group via the Telegram application. During the next 3 follow-up appointments, plaque index (PI), bleeding on probing (BOP), and gingival color and consistency were analyzed for each patient to assess their oral hygiene compliance during treatment.

Results. There was a statistically significant difference in Pl and BOP between the 2 groups at the $3^{\text {rd }}$ and $4^{\text {th }}$ appointments; however, gingival color and consistency did not differ significantly with regard to the manner of education $(p>0.05)$. Patient age did not have a considerable effect on the oral health status in either group $(p>0.05)$.

Conclusions. Teledentistry is an effective and efficient method to improve oral hygiene in patients undergoing fixed orthodontic treatment.

Keywords: orthodontics, telemedicine, mobile health, remote consultation 


\section{Introduction}

\section{What is teledentistry?}

Teledentistry is a type of telemedicine that uses information technology and telecommunication to provide access to dental care and consultation with specialists as well as to increase public awareness regarding oral health. ${ }^{1,2}$ This method can provide new opportunities for patients and clinicians to improve the quality of treatment. ${ }^{3}$ Moreover, teledentistry can be used as an approach for screening dental diseases, for patient education through conversation and videos, and patient referral for advanced dental treatment. ${ }^{4}$ Many studies have described the ease and reliability of this technology-based approach (with the aid of technologies like smartphones) ${ }^{2}$ as compared to the traditional methods. ${ }^{5}$ For example, in a study conducted by Alipour-Rocca et al., evaluating the ability of mothers to diagnose caries by means of teledentistry, it was concluded that mothers were able to diagnose caries through smartphone-based photographs. ${ }^{6}$ Teledentistry is not only used to increase access to dental care, but it can also be used for advanced dental education. ${ }^{5}$ Chen et al. stated in their study that teledentistry could extend care to people in distant locations, such as those in rural areas, with a reasonable cost and the aid of telecommunication technologies. ${ }^{7}$ Another study reported that teledentistry increased the quality of oral healthcare provided to children living in remote rural areas of California, USA. ${ }^{8}$

\section{Exacerbation of dental diseases associated with fixed orthodontic treatment}

Dental caries and periodontal problems may be exacerbated during fixed orthodontic treatment due to the lack of proper education. In this regard, one reason can be the lack of skilled practitioners in remote areas, and another can be the inability to refer patients to advanced dental healthcare systems due to geographic or financial limitations. In addition, patients undergoing fixed orthodontic treatment present changes in the oral cavity, such as a decrease in salivary $\mathrm{pH}$, the creation of additional food trap locations and increased levels of Streptococcus mutans. ${ }^{9}$ Nowadays, fixed orthodontic appliances are the most common devices for orthodontic treatment. ${ }^{10}$ It has been shown that the placement of bands and brackets leads to poor oral hygiene and an increased plaque index (PI). ${ }^{11}$ Since these appliances make controlling microbial plaque (the main cause of periodontal inflammation) more difficult, oral hygiene should be carefully monitored during the performance of orthodontic treatment. ${ }^{11,12}$ Plaque index and bleeding on probing (BOP) are the 2 main assessments used to interpret the oral hygiene status. ${ }^{13}$

\section{Objectives}

Based on the importance of oral hygiene in patients with fixed orthodontic appliances and the wide use of telecommunication via the Internet in healthcare education, this study aimed to evaluate the effect of teledentistry on the oral health status of patients undergoing fixed orthodontic treatment at the first 3 follow-up appointments.

\section{Material and methods}

This interventional study was designed with 60 participants selected from patients who were starting fixed orthodontic treatment in a dental clinic in Tehran, Iran, during the summer of 2019. Only patients with permanent dentition, class I dental and skeletal relationships with mild to moderate tooth size discrepancy, no need for extraction, no history of previous orthodontic treatment, no active medical or dental diseases, regular access to a smartphone, and willingness and informed consent to participate were included in this study.

All patients were treated using similar techniques and materials by a trained orthodontist. The participants were randomly divided into 2 groups while they were blinded to the group assignment. Each group was then divided into 2 subgroups based on the patients' age to investigate the effect of this parameter on the oral health status while performing orthodontic treatment. Patients aged $12-18$ years $(n=12)$ and patients older than 18 years $(n=18)$ were included in both trial arms.

\section{Treatment and intervention sequences}

Before banding and bonding, the patients underwent a session of oral hygiene measurements to obtain a PI of 0 . Then, they were examined in terms of BOP, gingival color (pink) and gingival consistency (firm) of the upper and lower arches (from right to left upper first molar and from left to right lower first molar). Of note, if there was any evidence of active periodontal disease, the participant was excluded.

The treatment sequence during the $1^{\text {st }}$ appointment consisted of banding all first molars, and bonding all upper and lower anterior and premolar teeth. Thereafter, a 0.012-inch nickel-titanium (Ni-Ti) wire was inserted; it was retied during the $2^{\text {nd }}$ appointment to relieve crowding more efficiently. During the $3^{\text {rd }}$ appointment, a 0.014-inch $\mathrm{Ni}-\mathrm{Ti}$ wire was put in place for the next 4 weeks.

For the control group, at the end of the $1^{\text {st }}$ appointment, an operator thoroughly explained the importance of oral hygiene during orthodontic treatment for about $5 \mathrm{~min}$. Thereafter, she used a dental model to instruct the participants precisely on the Bass brushing technique (the preferable technique for orthodontic patients) and the use of interdental brushes. She showed the replaceable tips of the brushes, 
which are used with no toothpaste, and are gently moved back and forth in the interdental spaces. She suggested using them in addition to regular tooth brushing twice a day - after lunch and before bedtime. Then, she recommended flossing at least once a day before bedtime with orthodontic dental floss due to its stiff head, which makes it easier to thread the floss under the archwire. She demonstrated threading plaque away from the gums. Finally, she explained the importance of using mouthwash and suggested rinsing 3 times a week at night, for 2-3 min each night.

In the teledentistry group, after the $1^{\text {st }}$ appointment, the participants received a video clip of a duration of $5 \mathrm{~min}$ and $7 \mathrm{~s}$ via the Telegram application. In the video, the same operator educated the participants about oral healthcare methods in a manner similar to the way the control group was instructed.

Plaque index, BOP, gingival color, and gingival consistency were recorded for each participant during the next 3 appointments at 4-week intervals. Finally, the collected data was entered into a checklist and subjected to statistical analysis.

\section{Ethical considerations}

The participants were fully informed and volunteered to participate in the study. Each participant's checklist information was completely confidential, and each participant was assigned a code. Ethical approval was obtained for this research from the Dental Research Center at Qom University of Medical Sciences in Iran (IR.MUQ. REC.1398.058).

\section{Statistical analysis}

The data was analyzed using the IBM SPSS Statistics for Windows software, v. 20.0 (IBM Corp., Armonk, USA). Differences in the mean PI and BOP scores between the control and intervention groups were evaluated using the independent $t$ test. Differences in the PI and BOP scores within each group were evaluated using the repeated analysis of variance (ANOVA). Afterward, the $\chi^{2}$ test was used to compare the mean changes in gingival color and consistency at each appointment. It was also used to evaluate the effect of patient age on the oral hygiene status during the course of treatment. Friedman's test was used to calculate the significance of changes in gingival color and consistency during the study. The level of statistical significance was set at 0.05 .

\section{Results}

\section{Plaque index and bleeding on probing}

As shown in Table 1 and Table 2, the mean PI and BOP scores were similar for both groups at the $2^{\text {nd }}$ appointment; however, the teledentistry group had decreased PI and BOP levels at the $3^{\text {rd }}$ and $4^{\text {th }}$ appointments. On the contrary, the control group showed increased levels of these 2 indices at the $3^{\text {rd }}$ and $4^{\text {th }}$ appointments.

Table 1. Comparison of the mean plaque index (PI) scores between the intervention and control groups at 3 follow-up visits

\begin{tabular}{|l|c|c|c|}
\hline Appointment & Group & PI & $p$-value \\
\hline \multirow{2}{*}{$2^{\text {nd }}$} & teledentistry & $36.12 \pm 19.40$ & \\
& control & $37.30 \pm 19.93$ & 0.820 \\
\multirow{2}{*}{$3^{\text {rd }}$} & teledentistry & $32.82 \pm 18.21$ & \\
& control & $46.45 \pm 22.11$ & $0.010^{*}$ \\
\multirow{2}{*}{$4^{\text {th }}$} & teledentistry & $24.91 \pm 12.76$ & \\
& control & $59.53 \pm 14.65$ & $0.000^{*}$ \\
\hline
\end{tabular}

* statistically significant.

Data presented as mean \pm standard deviation $(M \pm S D)$.

Table 2. Comparison of the mean bleeding on probing (BOP) scores between the intervention and control groups at 3 follow-up visits

\begin{tabular}{|l|c|c|c|}
\hline \multirow{2}{*}{ Appointment } & Group & $\begin{array}{c}\text { BOP } \\
{[\%]}\end{array}$ & $p$-value \\
\hline \multirow{2}{*}{$2^{\text {nd }}$} & teledentistry & $26.20 \pm 20.63$ & \multirow{2}{*}{0.680} \\
& control & $24.21 \pm 16.70$ & \\
\multirow{2}{*}{$3^{\text {rd }}$} & teledentistry & $23.40 \pm 15.73$ & \multirow{2}{*}{$0.020^{*}$} \\
& control & $36.00 \pm 23.24$ & \\
\multirow{2}{*}{$4^{\text {th }}$} & teledentistry & $19.47 \pm 14.21$ & \multirow{2}{*}{$0.004^{*}$} \\
& control & $67.67 \pm 85.64$ & \\
\hline
\end{tabular}

* statistically significant.

Data presented as $M \pm S D$.

\section{Gingival color}

As shown in Table 3, except for the $3^{\text {rd }}$ visit, during which there was no significant difference noticed between the 2 groups, the percentage of participants with coral pink gingivae was higher for the control group at the $2^{\text {nd }}$ appointment and higher for the teledentistry group at the $4^{\text {th }}$ appointment, with both differences statistically significant.

Table 3. Comparison of gingival color between the intervention and control groups at 3 follow-up visits

\begin{tabular}{|l|c|c|c|}
\multirow{2}{*}{ Appointment } & Group & $\begin{array}{c}\text { Participants with } \\
\text { coral pink gingivae } \\
(\%)\end{array}$ & $p$-value \\
& teledentistry & 33.3 & $0.020^{*}$ \\
\multirow{2}{*}{$2^{\text {nd }}$} & control & 63.3 & \\
& teledentistry & 53.3 & 0.440 \\
& control & 43.3 & $0.020^{*}$ \\
\hline \multirow{2}{*}{$4^{\text {th }}$} & teledentistry & 56.7 & \\
& control & 26.7 & \\
\hline
\end{tabular}

* statistically significant. 


\section{Gingival consistency}

This index was significantly different between the intervention and control groups at the $3^{\text {rd }}$ and $4^{\text {th }}$ appointments $(p \leq 0.05)$, and the differences were in favor of the teledentistry group. However, no significant difference was found at the $2^{\text {nd }}$ visit. Details are presented in Table 4.

According to the results of Friedman's test, there was no significant difference between the intervention and control groups in terms of gingival color and consistency during the study $(p>0.05)$.

Table 4. Comparison of gingival consistency between the intervention and control groups at 3 follow-up visits

\begin{tabular}{|l|c|c|c|}
\hline \multirow{2}{*}{ Appointment } & Group & $\begin{array}{c}\text { Participants with firm } \\
\text { and elastic gingivae }\end{array}$ & $p$-value \\
& teledentistry & 60.0 & 0.590 \\
& control & 66.7 & \\
\multirow{2}{*}{$3^{\text {rd }}$} & teledentistry & 86.7 & $0.037^{*}$ \\
& control & 63.3 & \\
$4^{\text {th }}$ & teledentistry & 80.0 & $0.001^{*}$ \\
\hline
\end{tabular}

* statistically significant.

\section{Effect of age}

In both the intervention and control trial arms, 40\% of the patients were between 12 and 18 years of age, and $60 \%$ were above 18 years of age. There was no significant difference between these 2 subgroups in terms of PI, BOP, gingival color, and gingival consistency during the study based on the $X^{2}$ test $(p>0.05)$.

\section{Discussion}

The evaluation of the efficacy of different educational techniques during dental treatment procedures may decrease the prevalence of such oral diseases as dental caries and periodontal problems. This study was conducted to investigate the efficacy of teledentistry as a relatively new telecommunication method for instructing patients undergoing fixed orthodontic treatment about oral hygiene. The results indicated that this method could have a considerable positive effect on the patients' oral health status during the first 3 months after starting treatment. The patients' knowledge about using new educational methods was improved, which might lead to the improved oral hygiene, and the reduced cost and pain burden for both the patients and the society.

In a previous study that evaluated the efficacy of a mobile phone-based application in teaching preventive dental care to the mothers of children under the age of 6 years, Hebbal et al. found that the mobile phone application significantly improved the mothers' knowledge about their children's oral health. ${ }^{14}$ Shah et al. assessed the improvement of oral health knowledge after exposure to an educational video in a hospital setting and reported a statistically significant increase in the knowledge scores. ${ }^{15}$ According to studies by Birang et al. ${ }^{16}$ and Darby and Walsh ${ }^{17}$ on using visual media to promote oral hygiene, visual media could improve oral health-related knowledge and practice, both in the short and long term. In addition, they could provide instructions in a faster and superior way. ${ }^{16,17}$

\section{Plaque index}

As a major indicator of the oral health status, PI decreased over the course of treatment in the teledentistry group. Notably, there was no significant difference in PI between the 2 groups at the $2^{\text {nd }}$ visit, but this index decreased in the teledentistry group at the $3^{\text {rd }}$ and $4^{\text {th }}$ appointments. Conversely, PI actually increased in the patients who had only been instructed in person. The reason for increased PI scores in the control group may be the fact that the participants had no access to an educational source like the video clip to be used as a guide at home. Therefore, they may have forgotten the instructions they had learned during their very first appointment. It is noteworthy that searching Internet sources is an easy way to access unlimited images, clips and websites with oral hygiene instructions during orthodontic treatment; however, many patients lack the time and/or interest to conduct a web search. As a result, deteriorating oral hygiene during fixed orthodontic treatment is a common consequence of using conventional education methods only. It should also be kept in mind that in the case of conventional education, the excessively frequent repetition of the topic may lead to a more negative attitude as well as decreased patient cooperation. ${ }^{18}$

Hebbal et al. evaluated the oral hygiene-related knowledge and PI scores of 12-year-old schoolchildren after health education with audiovisual aids as compared to control (with no education at all) and conventional education (chalk and blackboard) groups. ${ }^{14}$ After performing advanced intervention, the mean knowledge scores increased more in the trial group as compared to the conventional education and control groups. Although the mean PI scores decreased significantly in all 3 groups, the decrease was more evident when audiovisual aids were used as the educational method. ${ }^{14}$ Additionally, in accordance with the results of the abovementioned study, Albandar et al. found that the PI scores improved in a group of children who underwent a comprehensive education program in contrast to a control group. ${ }^{19}$ Other comparable studies also concluded that PI could be significantly improved after educational courses. ${ }^{20-23}$ While Najjari et al. reported lower PI scores after patient education, they found no statistically significant difference between intervention and 
control (with conventional education) groups in terms of this index. ${ }^{24}$ In contrast to our findings, Ghorbani reported increased PI levels in a group that underwent education with a movie. ${ }^{25}$ The author concluded that the conventional method of patient education is still the most logical and reliable way to improve the oral hygiene status. ${ }^{25}$ This difference may be related to the quality of instructions in the movie as well as the patients' desire and interest to learn from the educational movie.

\section{Bleeding on probing}

Similar to the PI scores, the mean scores of the BOP index were similar for the 2 groups at the $2^{\text {nd }}$ appointment, but then gradually decreased in the teledentistry group and increased in the control group. In line with these findings, 2 other studies showed decreased BOP levels after performing an intervention in the form of an educational video. ${ }^{24,26}$ However, in contrast to the results of the present investigation, there was no significant difference between the mean BOP scores of trial and control groups. ${ }^{24,26}$ Another study regarding BOP changes following an educational program (the WHO Health Promoting Schools project) showed a significant decrease in BOP levels after oral hygiene education was provided to schoolchildren. ${ }^{27}$

\section{Gingival color and consistency}

The results of this study indicate that some negative changes in gingival color and consistency occurred in both groups, but these changes were less prominent in the patients educated by means of teledentistry. This means that gingival health was preserved more in this group. In this regard, more investigations are needed to reach a definite conclusion.

Based on the results of this study, and since teledentistry is quite a new method for screening and improving the oral health status during fixed orthodontic treatment, more studies are required to establish definite guidelines and to make teledentistry a routine part of clinical orthodontic practice.

\section{Conclusions}

Teledentistry, which in this study meant sending an educational movie to patients using smartphones and a telecommunication application, can be considered a useful method to decrease plaque accumulation and gingival bleeding in patients undergoing fixed orthodontic treatment. The possibility of watching the educational movie more than once at home can act as a reminder, which may consequently improve the patient's cooperation. However, the results of this study should be interpreted with caution because of the limited number of investigations performed in this field.

\section{ORCID iDs}

Elahe Soltanmohamadi Borujeni (D) https://orcid.org/0000-0001-9358-0005 Fatemeh Sarshar (D) https://orcid.org/0000-0001-8846-2194 Maryam Nasiri (D) https://orcid.org/0000-0003-3256-8891 Samaneh Sarshar (D) https://orcid.org/0000-0002-7392-1551 Leila Jazi (D) https://orcid.org/0000-0002-3735-4764

\section{References}

1. Clark GT. Teledentistry: What is it now, and what will it be tomorrow? J Calif Dent Assoc. 2000;28(2):121-127.

2. Fricton $\mathrm{J}$, Chen $\mathrm{H}$. Using teledentistry to improve access to dental care for the underserved. Dent Clin North Am. 2009;53(3):537-248. doi:10.1016/j.cden.2009.03.005

3. Sachpazidis I. Image and medical data communication protocols for telemedicine and teleradiology (PhD thesis). Technical University of Darmstadt, Germany. 2008. https://tuprints.ulb.tu-darmstadt. de/1157/. Accessed January 1, 2021.

4. Ignatius E, Mäkelä K, Happonen RP, Perälä S. Teledentistry in dental specialist education in Finland. J Telemed Telecare. 2006;12(Suppl 3):46-49. doi:10.1258/135763306779379996

5. Mihailovic B, Miladinovic M, Vujicic B. Telemedicine in dentistry (teledentistry). In: Graschew G, ed. Advances in Telemedicine: Applications in Various Medical Disciplines and Geographical Areas. Rijeka, Croatia: InTech; 2011:215-230. doi:10.5772/14352

6. Alipour-Rocca L, Kudryk V, Morris T. TME3347: A teledentistry consultation system and continuing dental education via Internet. J Med Internet Res. 1999;1(Suppl 1):e110. doi:10.2196/jmir.1.suppl1.e110

7. Chen JW, Hobdell MH, Dunn K, Johnson KA, Zhang J. Teledentistry and its use in dental education. J Am Dent Assoc. 2003;134(3):342-346. doi:10.14219/jada.archive.2003.0164

8. Birnbach JM. The future of teledentistry. J Calif Dent Assoc. 2000;28(2):141-143.

9. Chandra G, Rao J, Singh K, Gupta K. Teledentistry in India: Time to deliver. J Educ Ethics Dent. 2012;2(2):61-64.

10. Estai $M$, Kanagasingam $Y$, Huang $B$, et al. The efficacy of remote screening for dental caries by mid-level dental providers using a mobile teledentistry model. Community Dent Oral Epidemiol. 2016;44(5):435-441. doi:10.1111/cdoe.12232

11. Elango KJ, Anandkrishnan N, Suresh A, lyer SK, Ramaiyer SK, Kuriakose MA. Mouth self-examination to improve oral cancer awareness and early detection in a high-risk population. Oral Oncol. 2011;47(7):620-624. doi:10.1016/j.oraloncology.2011.05.001

12. Cantekin K, Celikoglu M, Karadas M, Yildirim H, Erdem A. Effects of orthodontics treatment with fixed appliances on oral health status: A comprehensive study. J Dent Sci. 2011:6(4):235-238. doi:10.1016/j.jds.2011.09.010

13. Newman MG, Takei H, Klokkevold PR, Carranza FA. Carranza's Clinical Periodontology. $11^{\text {th }}$ ed. Philadelphia, USA: Saunders/Elsevier Health Sciences; 2011:14-872.

14. Hebbal M, Ankola AV, Vadavi D, Patel K. Evaluation of knowledge and plaque scores in school children before and after health education. Dent Res J (Isfhan). 2011;8(4):189-196. doi:10.4103/1735-3327.86036

15. Shah N, Mathur VP, Kathuria V, Gupta T. Effectiveness of an educational video in improving oral health knowledge in a hospital setting. Indian J Dent. 2016;7(2):70-75. doi:10.4103/0975-962X.184646

16. Birang R, Shakerian K, Yazdanpanah F, Nadimi M. The effects of education by visual media on oral health promotion of students. Arak Med Uni J. 2006;9(Special Issue):1-6.

17. Darby ML, Walsh MM. Dental hygiene: Theory and Practice. $3^{\text {rd }}$ ed. Philadelphia, USA: Saunders/Elsevier Health Sciences; 2009:70-71.

18. Laiho M, Honkala E, Nyyssönen V, Milen A. Three methods of oral health education in secondary schools. Eur J Oral Sci. 1993;101(6):422-427. doi:/10.1111/j.1600-0722.1993.tb01142.x

19. Albandar JM, Buischi YA, Mayer MP, Axelsson P. Long term effect of two preventive programs on the incidence of plaque and gingivitis in adolescents. J Periodontol. 1994;65(6):605-610. doi:10.1902/jop.1994.65.6.605

20. Ivanovic M, Lekic P. Transient effect of a short-term educational programme without prophylaxis on control of plaque and gingival inflammation in school children. J Clin Periodontol. 1996;23(8):750-757. doi:10.1111/j.1600-051X.1996.tb00605.x 
21. Glavind $L$, Zeuner $E$, Attström R. Oral hygiene instruction of adults by means of a self-instructional manual. J Clin Periodontol. 1981;8(3):165-176. doi:10.1111/j.1600-051x.1981.tb02028.x

22. Hetland L, Midtun N, Kristoffersen T. Effect of oral hygiene instructions given by paraprofessional personnel. Community Dent Oral Epidemiol. 1982;10(1):8-14. doi:10.1111/j.1600-0528.1982.tb00353.x

23. Söderholm G, Nobréus N, Attström R, Egelberg J. Teaching plaque control. I. A five-visit versus a two-visit program. J Clin Periodontol. 1982;9(3):203-213. doi:10.1111/j.1600-051x.1982.tb02060.x

24. Najari N, Mehidzadeh AH, Mehdizadeh M, Nodoushan ZJ, Mohammadbeigi A. A comparison between conventional and video methods of health education on improvement of oral health in elementary school students of Qomrood village, 2018 (Iran) [in Persian]. Qom Univ Med Sci J. 2019:13(5):25-32. doi:10.29252/qums.13.5.25

25. Ghorbani H. A comparison between the effect of conventional method and educational video on decreasing of the bacterial plaque in male students of primary school in Tehran in 2010 (MD thesis) [In Persian]. Shahid Beheshti University of Medical Sciences, Tehran, Iran. 2010.

26. Mofid R, Fathieh AR, Zamiri M, Eshghi B. Effect of two Iranian toothpastes (Nasim \& Pooneh) with and FDA-approved toothpaste (Crest Regular) to reduce gingivitis and plaque. JDS Shahid Beheshti Uni Med Sci.2005;23(2):304-312.

27. Petersen PE, Peng B, Tai B, Bian Z, Fan M. Effect of a school-based oral health education programme in Wuhan City, Peoples Republic of China. Int Dent J. 2004;54(1):33-41. doi:10.1111/j.1875-595x.2004.tb00250.x 